\title{
Witnessing Irreducible Dimension
}

\author{
Wan Cong, ${ }^{1}$ Yu Cai, ${ }^{1, *}$ Jean-Daniel Bancal, ${ }^{2}$ and Valerio Scarani ${ }^{1,3}$ \\ ${ }^{1}$ Centre for Quantum Technologies, National University of Singapore, 3 Science Drive 2, Singapore 117543, Singapore \\ ${ }^{2}$ Quantum Optics Theory Group, Department of Physics, University of Basel, Klingelbergstrasse 82, 4056 Basel, Switzerland \\ ${ }^{3}$ Department of Physics, National University of Singapore, 2 Science Drive 3, Singapore 117551, Singapore
}

(Received 15 November 2016; published 25 August 2017)

\begin{abstract}
The Hilbert space dimension of a quantum system is the most basic quantifier of its information content. Lower bounds on the dimension can be certified in a device-independent way, based only on observed statistics. We highlight that some such "dimension witnesses" capture only the presence of systems of some dimension, which in a sense is trivial, not the capacity of performing information processing on them, which is the point of experimental efforts to control high-dimensional systems. In order to capture this aspect, we introduce the notion of irreducible dimension of a quantum behavior. This dimension can be certified, and we provide a witness for irreducible dimension four.
\end{abstract}

DOI: 10.1103/PhysRevLett.119.080401

Introduction.-The Hilbert space dimension of a quantum system limits the amount of information that can be stored in it. The study of the power of fixed-dimensional systems is still topical today [1-3], and several experimental groups are implementing high-dimensional encoding and decoding of information [4-6]. Thus, for the purposes of quantum information processing, a proper certification of dimension should capture the users' capacity of exploiting that dimensionality, not just the dimension that "is there"-after all, the simplest particle or a single mode of any field are already infinite-dimensional. To put it with another example: two qubits are a ququart, but merely using a source of qubits twice does not guarantee the ability of processing the information of a ququart.

The past decade has seen the rise of device-independent certification: some important properties of quantum devices can be assessed by looking only at the observed input-output statistics. A lower bound on the Hilbert space dimension can be certified in this way. Such device-independent dimension witnesses (DIDWs) exist both as prepare-and-measure schemes [7,8] and as Bell-type schemes [3,9-11]. But which notion of dimension do they capture?

In this Letter, we first show that some existing DIDWs unfortunately capture only the dimension that is there. As such, they can certify high dimension while only sequential procedures are being implemented, like using a source of qubits several times and implementing classical feedforward. Having brought this issue to the fore, we define the dimension irreducible under sequential operations, or simply irreducible dimension, that can be inferred from the available observations. Finally, we introduce a witness of irreducible dimension four, that can be violated by a pair of ququarts and suitable measurements. This shows that one can obtain device-independent bounds for a notion of dimension more attuned to the needs of quantum information processing.
Sequential violation of dimension witnesses.-We focus on bipartite scenarios involving two noncommunicating parties, Alice and Bob. Alice's possible measurements are labeled by $x \in \mathcal{X}$, and her outcomes $a \in \mathcal{A}$. Bob's measurements are labeled by $y \in \mathcal{Y}$, and his outcomes $b \in \mathcal{B}$. Device-independent statements rely only on the family of probability distributions (the "behavior") $\mathcal{P}=\{P(a, b \mid x, y) \mid a \in \mathcal{A}, b \in \mathcal{B}, x \in \mathcal{X}, y \in \mathcal{Y}\}$.

As the title of the original Letter goes, the family of inequalities derived by Collins-Gisin-Linden-MassarPopescu (CGLMP) was meant to detect the nonlocality of high-dimensional quantum systems [12]. These inequalities, that have two inputs and $d$ outputs for both parties $(x, y \in\{0,1\}, a, b \in\{0,1,2, \ldots, d-1\})$, are therefore natural candidates for dimension witnessing: indeed, the first example of a DIDW was based on CGLMP $_{3}$ [9], and semi-device-independent witnessing of dimensions up to 20 was reported using the CGLMP family [4]. The DIDW character of $\mathrm{CGLMP}_{4}$ was studied more recently: it was found that a violation greater than $I_{4}=0.315$ lower bounds the dimension of the measured system to entangled ququarts [13].

One of the behaviors that exceeds the latter bound is $\mathcal{P}_{\text {MES4 }}$ obtained by taking the maximally entangled state (MES) of two ququarts $\left|\Phi_{4}\right\rangle_{A B}=\frac{1}{2}(|00\rangle+|11\rangle+|22\rangle+|33\rangle)$ and performing the local projective measurement on the bases $[14,15]$

$$
\begin{aligned}
& \left|a_{x}\right\rangle=\sum_{k=0}^{3} \frac{e^{i \frac{\pi}{2} a k}}{2} e^{i k \alpha_{x}}|k\rangle, \\
& \left|b_{y}\right\rangle=\sum_{k=0}^{3} \frac{e^{-i \frac{\pi}{2} b k}}{2} e^{i k \beta_{y}}|k\rangle,
\end{aligned}
$$

with $\alpha_{0}=0, \alpha_{1}=(\pi / 4), \beta_{0}=-(\pi / 8)$, and $\beta_{1}=(\pi / 8)$. Indeed, one would find $I\left(\mathcal{P}_{\text {MES4 }}\right) \approx 0.336$ [13]. 
Consider now the following encoding of a ququart into two qubits:

$$
\begin{aligned}
|0\rangle & \mapsto|0\rangle \otimes|0\rangle, & |1\rangle & \mapsto|0\rangle \otimes|1\rangle, \\
|2\rangle & \mapsto|1\rangle \otimes|0\rangle, & |3\rangle & \mapsto|1\rangle \otimes|1\rangle .
\end{aligned}
$$

If both Alice and Bob perform this encoding, it is well known that the MES is mapped to the product of two two-qubit MESs: $\left|\Phi_{4}\right\rangle_{A B} \mapsto\left|\phi^{+}\right\rangle_{A_{1} B_{1}} \otimes\left|\phi^{+}\right\rangle_{A_{2} B_{2}}$, with $\left|\phi^{+}\right\rangle=(1 / \sqrt{2})(|00\rangle+|11\rangle)$. But here, also the optimal measurement bases (1) and (2) factor as sequential measurements. For Alice's (Bob's being analog), it reads

$$
\begin{aligned}
&\left|0_{x}\right\rangle_{A} \mapsto\left|+{ }_{2 \alpha_{x}}\right\rangle_{A_{1}} \otimes\left|+{ }_{\alpha_{x}}\right\rangle_{A_{2}}, \\
&\left|1_{x}\right\rangle_{A} \mapsto\left|-2 \alpha_{x}\right\rangle_{A_{1}} \otimes\left|+{ }_{\alpha_{x}+\frac{\pi}{2}}\right\rangle_{A_{2}}, \\
&\left|2_{x}\right\rangle_{A} \mapsto\left|+{ }_{2 \alpha_{x}}\right\rangle_{A_{1}} \otimes\left|--_{\alpha_{x}}\right\rangle_{A_{2}}, \\
&\left|3_{x}\right\rangle_{A} \mapsto\left|-2 \alpha_{x}\right\rangle_{A_{1}} \otimes\left|-{ }_{\alpha_{x}+\frac{\pi}{2}}\right\rangle_{A_{2}},
\end{aligned}
$$

where $\left| \pm_{\varphi}\right\rangle=\frac{1}{\sqrt{2}}\left(|0\rangle \pm e^{i \varphi}|1\rangle\right)$ are the eigenstates of $\sigma_{\varphi}=\cos \varphi \sigma_{x}+\sin \varphi \sigma_{y}$. Explicitly, this means that one can produce the behavior $\mathcal{P}_{\text {MES4 }}$ by the following sequential strategy: (i) The source sends out a pair of maximally entangled qubits. Given $x$, Alice measures her qubit in the basis $\left| \pm_{2 \alpha_{x}}\right\rangle$; given $y$, Bob measures his qubit in the basis $\left| \pm_{2 \beta_{y}}\right\rangle$. (ii) Later, the source sends out a second pair of qubits. If Alice obtained the outcome + in her first measurement, she now measures the second qubit in the basis $\left| \pm_{\alpha_{x}}\right\rangle$; if she obtained - , in the basis $\left| \pm_{\alpha_{x}+\frac{\pi}{2}}\right\rangle$. Bob follows the analog procedure.

In particular, one ends up certifying dimension four on both sides, where only a two-qubit source (admittedly used twice), single-qubit local manipulations, and classical feedforward were implemented. For a sequential violation of the CGLMP inequality when the number of outcomes $d$ that is a power of two, see also [16]. In Supplemental Material [17], Sec. I, we show that the qutrit dimension witness based on CGLMP $_{3}$ [9] may also be violated using solely two-qubit sources and sequential single-qubit measurements.

Hoping to better capture the experimental effort in proper high-dimensional quantum experiments rather than sequential procedures, we introduce the notion of dimension irreducible by sequential operations, or simply irreducible dimension, of a quantum behavior; and we provide an example of a DIDW that certifies irreducible dimension four.

Correlations from sequential d-dimensional systems.Let us define a sequential $d$-dimensional model as consisting of (i) $d$-dimensional sources (dimensionality), (ii) operations and measurements performed sequentially on each $d$-dimensional system, possibly feeding forward the measurement outcomes (sequentiality), and (iii) arbitrary local classical processing and shared randomness.

We then say that a behavior is sequential $d$-dimensional compatible if it can be obtained with each involved party individually following a sequential $d$-dimensional model. The set of sequential $d$-dimensional compatible behaviors can be seen to be the closure under wiring $[19,20]$ of the set of $d$-dimensional quantum correlations $[3,21]$. Unfortunately, few explicit sets are known to be closed under wiring, and in general it is not known how to characterize the closure under wiring of a given set $[19,20]$.

The smallest $d$ such that the behavior is sequentially $d$ compatible is called the dimension irreducible by sequential operations, or simply the irreducible dimension of the behavior. Every behavior that can be simulated with classical resources, including those describing prepareand-measure schemes, has irreducible dimension 1. Just as for entanglement or nonlocality, nontrivial irreducible dimension must necessarily involve more than one party. The behavior $\mathcal{P}_{\text {MES4 }}$ has irreducible dimension 2 .

Given the previous example, one may fear that any probability distribution can be achieved by combining sufficiently many sequential measurements on qubit systems, rendering irreducible dimension witnessing a somewhat trivial exercise also in the quantum case. Fortunately, this is not the case: there exist distributions that lower bound the dimension of the involved devices to more than two even when sequential strategies are considered.

A quantum behavior with irreducible dimension four.A witness for irreducible dimension four must rule out sequential measurements on consecutive qubits and qutrits. We do not know how to express all of these constraints as a function of the observed probability distributions $P(a, b \mid x, y)$ in simple terms. In order to construct an example, we notice that an entangled measurement, one whose eigenvectors are entangled states, cannot be sequential, since an entangled measurement cannot be achieved even with bidirectional classical communication. Besides, the minimal dimension to have entanglement is $d=4$ (two qubits). Thus, certification of such a measurement guarantees that a four-dimensional nonsequential operation is performed.

The possibility of certifying entangled measurements was demonstrated in the entanglement-swapping configuration, i.e., in a tripartite scenario, either assuming knowledge of the dimensions [22] or in the fully deviceindependent setting [23]. Exploiting a recent result on selftesting [24], we construct an explicit behavior for a bipartite scenario, such that one of Bob's measurements can be certified to be entangled.

Our behavior, denoted $\mathcal{P}_{\mathrm{BSM}}=\{P(a, b \mid x, y): a, b$, $x \in\{0,1,2,3\} ; y \in\{0,1,2,3,4\}\}$, uses four measurements for Alice and five measurements for Bob, each having four possible outcomes. The entangled measurement will be $y=4$. We need the other measurements to 
first establish that both Alice's and Bob's systems are composed of two subsystems in a local separable state, which cannot be assumed a priori in a device-independent setting.

Let us first leave $y=4$ aside. The parties label each of the four-valued input and outcomes $a, b, x, y \in\{0,1,2,3\}$ as two bits: $c=2 c_{1}+c_{2} \rightarrow\left(c_{1}, c_{2}\right)$. If

$P(a, b \mid x, y)=\prod_{i=1,2} P_{2 \sqrt{2}}\left(a_{i}, b_{i} \mid x_{i}, y_{i}\right), x, y \in\{0,1,2,3\}$,

where $P_{2 \sqrt{2}}$ is the unique probability point that violates maximally the Clauser-Horne-Shimony-Holt (CHSH) inequality, then the state shared between Alice and Bob is self-tested to the product $\left|\phi^{+}\right\rangle_{A_{1} B_{1}} \otimes\left|\phi^{+}\right\rangle_{A_{2} B_{2}}$ of two maximally entangled two-qubit states [24]. Thus, (4) certifies that there are indeed two subsystems in a separable state, both on Alice's side (denoted $A_{1}$ and $A_{2}$ ) and on Bob's $\left(B_{1}\right.$ and $\left.B_{2}\right)$. In this situation, if $A_{1}$ and $A_{2}$ are found entangled conditioned on the outcome of $y=4$, then $y=4$ must be an entangled measurement on $B_{1}$ and $B_{2}$.

In order to test entanglement on Alice's side, we need suitable measurements, local on her subsystems - and we have got them already. Indeed, it is an important feature of self-testing that not only the state but also the measurements on the subsystems are self-tested as the optimal measurements for $\left|\phi^{+}\right\rangle$to violate the $\mathrm{CHSH}$ inequality [24]. Thus, we know that Alice's measurements are $\sigma_{z} \otimes \sigma_{z}, \sigma_{x} \otimes \sigma_{z}, \sigma_{z} \otimes \sigma_{x}$, and $\sigma_{x} \otimes \sigma_{x}$, up to local isometries.

Now we have a simple recipe to finish the construction of $\mathcal{P}_{\mathrm{BSM}}$ : for the measurement labeled $y=4$, we choose the Bell-state measurement (BSM) on $B_{1}$ and $B_{2}$, which prepares $A_{1}$ and $A_{2}$ in states that violate $\mathrm{CHSH}$ maximally for those measurements of Alice. Thus, we will have

$$
P(a, b \mid x, 4)=\frac{1}{4} P_{2 \sqrt{2}, b}\left(a_{1}, a_{2} \mid x_{1}, x_{2}\right),
$$

since, as explained in Supplemental Material, one must use a different CHSH expression for each value of Bob's outcome $b$. All the details are given in Supplemental Material [17], Sec. II.

In summary, if one observes $\mathcal{P}_{\text {BSM }}$ defined by (4) and (5), then the density matrix and measurement operators are acting locally on $\mathbb{C}^{d}$ with $d \geq 4$, and the statistics cannot be reproduced by Alice and/or Bob sequentially measuring several smaller-dimensional (qubit or qutrit) sources in their respective labs. In short, the behavior $\mathcal{P}_{\mathrm{BSM}}$ has irreducible dimension greater than or equal to four.

A witness of irreducible dimension four.-In the previous section, $\mathcal{P}_{\mathrm{BSM}}$ is just one behavior, i.e., a single point in probability space: as such, it will never be observed exactly. In order to have a robust witness of irreducible dimension four, we need to demonstrate that, when the observed probability point is not exactly $\mathcal{P}_{\mathrm{BSM}}$, one party is still performing an entangled measurement.

It is clear that there is a large room for robustness in the $P(a, b \mid x, 4)$, i.e., in the choice of the entangled measurement itself: any behavior that shows a violation of $\mathrm{CHSH}$ (not necessarily maximal) for at least one value of $b$ would do. It is less easy to relax the self-testing part (4), because one immediately loses the sharp conclusion on the existence of subsystems. In the absence of well-defined subsystems, the notion of entangled measurement becomes blurred.

In order to estimate the robustness of the criterion, we presume the existence of subsystems $B_{1}$ and $B_{2}$ on Bob's side and assume that the projectors of the first four measurements of Bob are of the form

$$
\Pi_{b \mid y}^{B}=\Pi_{b_{1} \mid y_{1}}^{B_{1}} \otimes \Pi_{b_{2} \mid y_{2}}^{B_{2}},
$$

where $\left\{\Pi_{b_{1} \mid y_{1}}^{B_{1}}\right\}_{b_{1}, y_{1}=0,1}$ is a two-outcome projective measurement on $B_{1},\left\{\Pi_{b_{2} \mid y_{2}}^{B_{2}}\right\}_{b_{2}, y_{2}=0,1}$ is a two-outcome projective measurement on $B_{2}$, and with $c=2 c_{1}+c_{2}$ as before. Physically, this is equivalent to Bob measuring $B_{1}$ and $B_{2}$ independently and concatenating the two outcomes into one outcome string.

Under this assumption, we now demonstrate the robustness of the certification of entangled measurements and hence of irreducible dimension. For this, we make use of the SWAP technique $[25,26]$. A so-called SWAP operator, defined in terms of the parties' measurements, is used to exchange a particular part of the measured system with an auxiliary system of trusted dimension. Any linear function of the resulting quantum state can then be bounded over all quantum realizations through the Navascués-Pironio-Acín (NPA) hierarchy of semidefinite programing (SDP) [27].

Here, we thus consider two external qubits registers for Bob together with two qubit SWAP operators [25], each operator swapping subsystem $B_{1}$ (respectively, $B_{2}$ ) with one of the external qubit registers. The resulting doubleSWAP operator on Bob's system can be expressed in terms of Bob's measurement operators $\Pi_{b \mid y}^{B}$ as

$$
\mathcal{S}_{B B^{\prime}}|i, j\rangle_{B^{\prime}}=\sum_{k, l=0}^{1}|k, l\rangle_{B^{\prime}} X_{k, l} \Pi_{f(i, j, k, l) \mid 0}^{B} X_{i, j},
$$

where $f(i, j, k, l)=2(i \oplus k)+(j \oplus l), \quad X_{i, j}=$ $\sum_{k=0}^{3}(-1)^{j k+i\lfloor k / 2\rfloor} \Pi_{k \mid 3}^{B}$, and $\oplus$ is the sum modulo 2 (cf. Supplemental Material [17], Sec. III).

We then estimate whether Bob's fifth measurement is entangled by computing

$$
F=\frac{1}{4} \sum_{i=0}^{3} \operatorname{Tr}\left[\Pi_{i \mid 4}^{B} \mathcal{S}_{B B^{\prime}}\left(\rho_{A B} \otimes\left|\varphi_{i}\right\rangle_{B^{\prime}}\left\langle\varphi_{i}\right|\right) \mathcal{S}_{B B^{\prime}}^{\dagger}\right],
$$




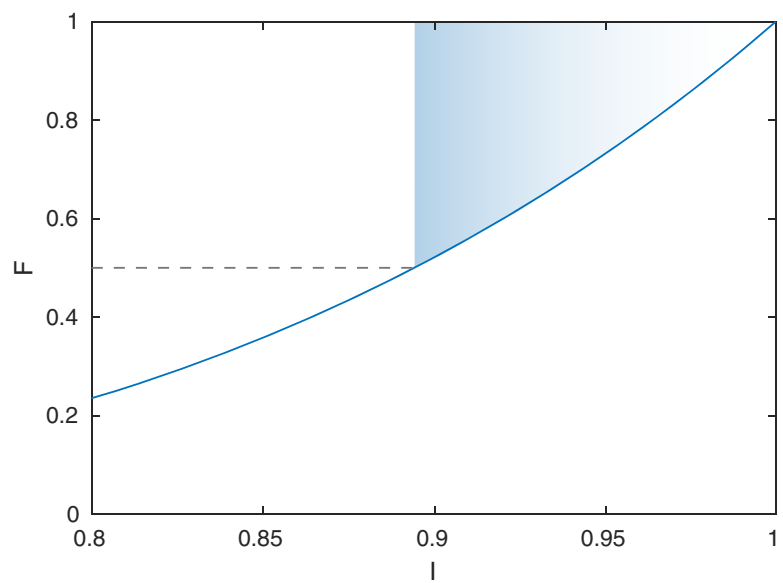

FIG. 1. Lower bound on the fidelity $F$ as a function of the violation of the Bell inequality $I \leq 0$. The shaded area highlights values of $I$ above $I^{*} \simeq 0.8942$, which guarantee that $F>1 / 2$. The maximal quantum violation of $I$ is $I=1$.

where $\left|\varphi_{i}\right\rangle=\sum_{j}(-1)^{i j}|j\rangle \otimes|\lfloor i / 2\rfloor \oplus j\rangle$ are the four Bell states. This expression can be understood as follows: the SWAP operator places a maximally entangled state in $B_{1} \otimes B_{2}$, after which one checks how close $\Pi_{i \mid 4}^{B}$ is to $\left|\varphi_{i}\right\rangle\left\langle\varphi_{i}\right|$. In Supplemental Material [17], Sec. III, we show that this fidelity is related to the fidelity of an entanglement swapping protocol that used Bob's last measurement to entangle two remote qubits; when $F>1 / 2$, at least one of Bob's measurement operators must be entangled. This conclusion is contingent on the assumption (6) made on Bob's system, which ensures that the SWAP operator (7) factorizes according to $\mathcal{S}=\mathcal{S}_{I} \otimes \mathcal{S}_{I I}$, where $\mathcal{S}_{I}$ acts on $B_{1}$ and the auxiliary system $B_{1}^{\prime}$ and $\mathcal{S}_{I I}$ acts on $B_{2}$ and $B_{2}^{\prime}$. An example showing the importance of this assumption for the presented argument is given in Supplemental Material [17], Sec. III.

To bound the quantity $F$ over all possible quantum realizations which are compatible with some behavior $\mathcal{P}$, we constructed an SDP matrix of size $390 \times 390$ corresponding to a relaxation of the NPA hierarchy [27]. We then minimized $F$ over all such matrices which are compatible with the chosen quantum behavior. For the sake of an example, let us consider the behavior obtained with a perfect implementation of the measurements on the tensor product of two two-qubit Werner states $\left[V\left|\phi^{+}\right\rangle\left\langle\phi^{+}\right|+(1-V) \square / 4\right]^{\otimes 2}$, resulting in a noisy version of $\mathcal{P}_{\text {BSM }}$. We find $F>1 / 2$ for $V \gtrsim 0.987$ (cf. Supplemental Material [17], Sec. IV). The corresponding dual SDP program provides a certificate for this conclusion in the form of a bipartite Bell inequality. This conclusion is readily confirmed by computing the minimal fidelity $F$ which is compatible with some violation of the inequality $I$ (cf. Fig. 1).

Conclusion.-In this Letter, we pointed out that some device-independent dimension witnesses can be violated with lower-dimensional systems and sequential measurements on them. This somehow defeats the operational goal of these witnesses, which is not simply to prove that some dimensionality is there but rather to certify that one can do quantum information processing. The same concern should be raised also for non-DI dimension witnesses: for instance, the lower bounds of Ref. [28] are multiplicative for product correlations, so by just using a qubit source $n$ times they certify dimension $2^{n}$.

Then we showed that this obstacle can be overcome: it is possible to construct witnesses that capture a more appropriate notion of dimension, namely, what we called the irreducible dimension of a quantum behavior. This solution was based on an example of entangled measurements certification. From now onwards, in the presence of a dimension witness, it will be important to check which irreducible dimension it certifies.

Some problems remain open. The robustness of our criterion was proved under some additional assumptions, because we have not found a way of identifying subsystems in a device-independent setting. Alternatively, one may think of approaches that are based on different criteria. It would also be interesting to investigate the case where the sequentiality assumption that we used here is removed. Ruling out that low-dimensional states and operations can be responsible for some observed behavior independently of the way in which these resources are combined would then lead to witnessing behaviors with genuine dimension $d$. A similar problem in the context of entanglement theory with characterized devices was recently considered by Kraft et al. [29]. In the ideal case, this work can be made device independent by using self-testing [30]. Independently of these questions, it would also be interesting to obtain a compact characterization of the statistics achievable with sequential measurements. This might provide an alternative approach to study the closureunder-wiring set of correlations.

We thank Nicolas Brunner, Jiangbin Gong, Otfried Guehne, and Miguel Navascués for feedback and discussions. This research is supported by the Singapore Ministry of Education Academic Research Fund Tier 3 (Grant No. MOE2012-T3-1-009); by the National Research Fund and the Ministry of Education, Singapore, under the Research Centres of Excellence program; and by the Swiss National Science Foundation (SNSF), through the NCCR QSIT and Grant No. PP00P2-150579.

*caiyu01@gmail.com

[1] J. I. de Vicente, Phys. Rev. A 95, 012340 (2017).

[2] P. E. Frenkel and M. Weiner, Commun. Math. Phys. 340, 563 (2015).

[3] M. Navascués and T. Vértesi, Phys. Rev. Lett. 115, 020501 (2015).

[4] A. C. Dada, J. Leach, G. S. Buller, M. J. Padgett, and E. Andersson, Nat. Phys. 7, 677 (2011). 
[5] M. Malik, M. Erhard, M. Huber, M. Krenn, R. Fickler, and A. Zeilinger, Nat. Photonics 10, 248 (2016).

[6] N. T. Islam, A. Aragoneses, A. Lezama, J. Kim, and D. J. Gauthier, Phys. Rev. Applied 7, 044010 (2017).

[7] N. Brunner, M. Navascués, and T. Vértesi, Phys. Rev. Lett. 110, 150501 (2013).

[8] R. Gallego, N. Brunner, C. Hadley, and A. Acín, Phys. Rev. Lett. 105, 230501 (2010).

[9] N. Brunner, S. Pironio, A. Acin, N. Gisin, A. A. Méthot, and V. Scarani, Phys. Rev. Lett. 100, 210503 (2008).

[10] K. F. Pál and T. Vértesi, Phys. Rev. A 77, 042105 (2008).

[11] T. Vértesi and K. F. Pál, Phys. Rev. A 79, 042106 (2009).

[12] D. Collins, N. Gisin, N. Linden, S. Massar, and S. Popescu, Phys. Rev. Lett. 88, 040404 (2002).

[13] Y. Cai, J.-D. Bancal, J. Romero, and V. Scarani, J. Phys. A 49, 305301 (2016).

[14] A. Acín, T. Durt, N. Gisin, and J. I. Latorre, Phys. Rev. A 65, 052325 (2002).

[15] S. Zohren and R. D. Gill, Phys. Rev. Lett. 100, 120406 (2008).

[16] H.-P. Lo, C.-M. Li, A. Yabushita, Y.-N. Chen, C.-W. Luo, and T. Kobayashi, Sci. Rep. 6, 22088 (2016).

[17] See Supplemental Material at http://link.aps.org/ supplemental/10.1103/PhysRevLett.119.080401for an example of a violation of qutrit dimension witness, explicit construction of $\mathcal{P}_{\mathrm{BSM}}$, and details in the robust certification of entangled measurements, which includes Refs. [18].
[18] D. Collins and N. Gisin, J. Phys. A 37, 1775 (2004).

[19] J. Allcock, N. Brunner, N. Linden, S. Popescu, P. Skrzypczyk, and T. Vértesi, Phys. Rev. A 80, 062107 (2009).

[20] B. Lang, T. Vértesi, and M. Navascués, J. Phys. A 47, 424029 (2014).

[21] M. Navascués, A. Feix, M. Araújo, and T. Vértesi, Phys. Rev. A 92, 042117 (2015).

[22] T. Vértesi and M. Navascués, Phys. Rev. A 83, 062112 (2011).

[23] R. Rabelo, M. Ho, D. Cavalcanti, N. Brunner, and V. Scarani, Phys. Rev. Lett. 107, 050502 (2011).

[24] X. Wu, J.-D. Bancal, M. McKague, and V. Scarani, Phys. Rev. A 93, 062121 (2016).

[25] T. H. Yang, T. Vértesi, J.-D. Bancal, V. Scarani, and M. Navascués, Phys. Rev. Lett. 113, 040401 (2014).

[26] J.-D. Bancal, M. Navascués, V. Scarani, T. Vértesi, and T. H. Yang, Phys. Rev. A 91, 022115 (2015).

[27] M. Navascués, S. Pironio, and A. Acín, Phys. Rev. Lett. 98, 010401 (2007); New J. Phys. 10, 073013 (2008).

[28] J. Sikora, A. Varvitsiotis, and Z. Wei, Phys. Rev. Lett. 117, 060401 (2016).

[29] T. Kraft, C. Ritz, N. Brunner, M. Huber, and O. Gühne, arXiv:1707.01050.

[30] A. Coladangelo, K. T. Goh, and V. Scarani, Nat. Commun. 8, 15485 (2017). 